\title{
Designing an Electronic Medical Case Simulator for Health Professional Education
}

\author{
Ronald S. Joe* \\ School of Health Information Science \\ University of Victoria \\ Victoria, British Columbia, Canada \\ E-mail: ronjoe@shaw.ca
}

\section{Anthony Otto}

School of Health Information Science

University of Victoria

Victoria, British Columbia, Canada

E-mail: agotto@telus.net

\section{Elizabeth Borycki}

School of Health Information Science

University of Victoria

Victoria, British Columbia, Canada

E-mail: emb@uvic.ca

*Corresponding author

\begin{abstract}
This paper describes an implementation of an Electronic Medical Record (EMR) which has been adapted for the purposes of teaching health professional students, including medical and nursing students. Off-the-shelf EMR software, while suited for physicians in practice settings does not completely satisfy the needs of these students and educators. There are many unique requirements of a teaching EMR compared to one used in a production environment. This paper describes the specific architecture and unique features of an EMR that was employed in the University of British Columbia Medical School teaching program in December, 2007 with 200 participating medical students distributed across three physical sites in the Province of British Columbia.
\end{abstract}

Keywords: Medical case simulator; Electronic medical records; Health professional education; Simulations

Biographical notes: Ronald Joe is a practicing physician who has developed a range of electronic health record applications for use in practice and for education. He holds a Masters in Health Information Science from the University of Victoria.

Anthony Otto is also a practicing physician who has extensive experience in the area of electronic health record systems. He also holds a Masters degree in Health Information Science from the University of Victoria. 
Elizabeth Borycki is an Assistant Professor at the School of Health Information Science at the University of Victoria. She has extensive expertise in the area of health informatics and holds a $\mathrm{PhD}$ from the University of Toronto.

\section{Introduction}

In Canada, the Federal Government through its initial investment of 1.2 billion dollars formed Canada Health Infoway (CHI) in 2000. Its mandate to accelerate and to enable information technology in healthcare, has gained considerable momentum over the last several years. Its focus is around creating a pan-Canadian electronic health record system (EHR). This goal, if achieved, would allow for electronic entry and retrieval of patient data by physicians and healthcare professionals across Canada. Its original specific objective was to achieve a 50\% adoption rate across the country by 2009 (Canada Health Infoway, 2006). The EHR would connect up patient data from different sources, including the electronic medical record (EMR) which is the electronic patient record in the physician's office or clinic. The benefits of achieving this would be a reduction in number of patient records being kept on paper and the potential for greatly modernizing and streamlining healthcare processes across Canada. CHI partnered primarily with the provincial and territorial jurisdictions in a co-funding relationship sharing in the cost of this venture. Its initial mandate was not to deliver EMRs to the desktops of every physician; however, many provinces and territories have taken their own initiative to fund this dissemination of this key information technology.

The British Columbia government in a deal ratified by the British Columbia Medical Association in March 2006 negotiated substantial funding to provide EMRs in physicians' offices (PITO, 2007). Such funding was an integral part of the province's investment in the eHealth framework (eHealth Steering Committee, 2005; Ministry of Health of BC, 2006) as a whole. By enabling information sharing, avoiding duplicate or unnecessary tests, and medical errors (Kohn, Corrigan, \& Donaldson, 2000; Bates et al., 1999), clinical guideline adherence and decision support, it is hoped that improvements in the quality of care is achieved in a cost effective and sustainable manner (Romanow, 2002) through the use of an EMR.

With its high level of commitment in eHealth, the British Columbia government also allocated specific funding to be utilized for teaching our future health care providers the new tools of the trade. The University of Victoria (UVIC) in British Columbia has considerable expertise in health informatics, was given the opportunity to architect the introduction of EMRs into the University of British Columbia (UBC) medical teaching program for the final year students.

Currently, the use of EMRs in education is in its infancy. A recent review found fewer than 50 articles with evidence of their use in medical education (Keenan, Nguyen, $\&$ Srinivasan, 2006). Some implementations have focused on teaching fundamental concepts only (Speedie, \& Niewoehner, 2003), whereas others have attempted to integrate an EMR in a family practice setting (Zelnick, \& Nelson, 2002). There are no implementations known which introduce an EMR in a didactic problem based teaching program.

A review of the current commercial and non-commercial systems revealed very few options. Non-commercial systems lacked basic functionality and commercial systems were too costly and configured to operate in actual practice settings only. All systems 
lacked the ability to create a standardized patient from which all students could work. Additional requirements of an educational EMR included comparing each individual student's actions, is not be possible with off the shelf solutions.

In this paper we describe our work in creating a unique EMR simulator for health professional education. The simulator was designed to have the features of a real working EMR (and was designed based on a system in use in medical practice), plus additional simulation-based features that allow for use and customization of the software for health professional education. The simulator has the ability to present medical students with patient cases within an electronic health record environment.

\section{The Requirements}

The authors, in May 2007 initiated discussions for designing an EMR to suit the specific needs of teaching. The specific requirements were determined through teleconference meetings with the faculty staff at both the UVIC and UBC. It was agreed that the basic requirement of an educational EMR should have a generic look and feel not too dissimilar to that seen in commercial systems in existence. In addition to the basic requirement, there is a need for customizing an EMR to have specific student and educator roles.

The educator's requirements would include: an ability to build a standardized patient case that contains information about a specific hypothetical patient case, a clinical scenario that describes the course of illness of the standardized patient, and the ability to view and compare each student's interaction with the case. Whereas, the student's requirements would include: access to their private workspace in which to store the standard case, and the ability to store of any subsequent changes to the case inputted by the student.

While these requirements are natural expectations in an educational setting, commercial systems are not designed to hold multiple copies of the same patient to be accessible and for changes to be made by different students. Demonstration modes in existing EMRs may be the closest to this ability, however, this mode cannot track and store changes made by each individual student on a single patient case.

The cost and the ancillary resources needed to customize and implement a commercial system to add student and educator interactive roles would be prohibitive. The timeframe for the roll out of the EMR software was also short.

The authors therefore, decided to customize their own system. The basic system called the Anthrologix EMR core engine is a generic object orientated medical record keeping system written in the Microsoft .NET framework. The EMR core engine uses modern object orientated structures. This is in contrast to the majority of EMR systems in existence today using older software systems or components. These older systems are difficult to change and if it were possible would require development of a separate customized software solution.

The advantage of a well organized and flexible object oriented EMR data structure in the Anthrologix core leads itself to making the necessary changes easily. 


\section{The EMR Case}

The teaching was conducted during the week of the 10th of December, 2007. The hands on use of the EMR was integrated into the problem based learning series in the final year medical school program (Borycki et al., 2009). The EMR case comprised of a hypothetical patient named Tom Miller who is a 45 year old male presenting with a history of back pain experiencing a complicated course eventually requiring surgery. An introductory course consisting of basic information relating to EMRs preceded a demonstration of the software. During the course of the week, small groups were formed sharing a laptop with the EMR software installed to give the student hands on experience. Some of the functions suggested included entering an encounter, disease coding, obtaining a consultation from an orthopaedic surgeon and retrieving a hospital discharge summary. There was also an opportunity to discuss issues exposed in a shared record to include privacy and security issues.

\section{The Implementation}

The specifics of the software implementation at the initial meetings was to deploy a system which consisted of a centralized database and clients would log onto the EMR, or what is commonly know as a client - server. Unfortunately, as the technical requirements were being fleshed out, there were insurmountable barriers to this type of implementation. The UBC course was being taught at three physical sites and not all of students had equal access to computers from which to $\log$ in and use the EMR. Some students were located at hospital sites as they were part of clinical electives. Hospitals had restricted access to external applications including the Windows Remote Desktop application which was the intended means of accessing the central database. The EMR was application based and not web based, so a browser solution was not possible. The client - server approach was therefore abandoned and local installation into laptops was decided upon. A supplemental option of downloading the software into the student's home computer was also offered through the UBC educational portal. This UBC educational portal was also used to disseminate the user manual for the EMR.

\section{The Software}

The preexisting Anthrologix EMR graphical user interface (Fig. 1) was used with minor modifications. This included a tab to expose a control panel for students and another for educators (Fig. 5). The panel for the students would be the means within the software to subscribe to a preset case and to keep track of all cases subscribed to. The panel for educators would be the means to create one or many cases in addition to setting up the conditions in which the objects of the patient case would appear.

To enable most of new functions of an educational EMR new student and educator modes (Fig. 2) needed to be added to the existing user object in the EMR. The user object of the EMR contains all information about users of the system. The contents of this object also determine access privileges. In this case, an additional field was added to the user object to hold the user mode, whether it is: an educator, a student, or a normal or default mode. The normal or default mode would to allow the EMR to function as it was originally designed or for a typical physician's office. Mode selection, therefore, allows a single application to handle the needs of a typical production system as well as those needed for education purposes. This refers back to most commercial systems needing to produce a customized solution that is a separate application. 


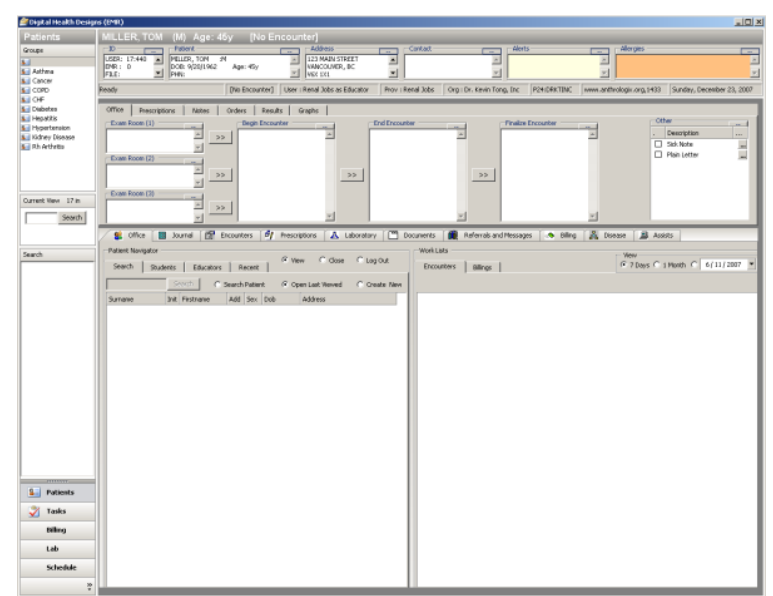

Figure 1. Screen shot of the EMR

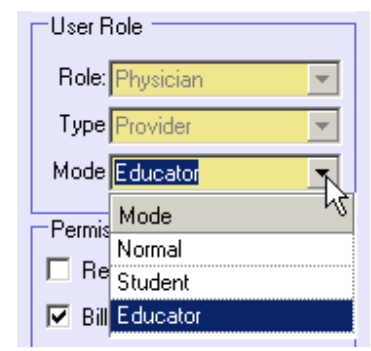

Figure 2. Setting the user modes of the EMR

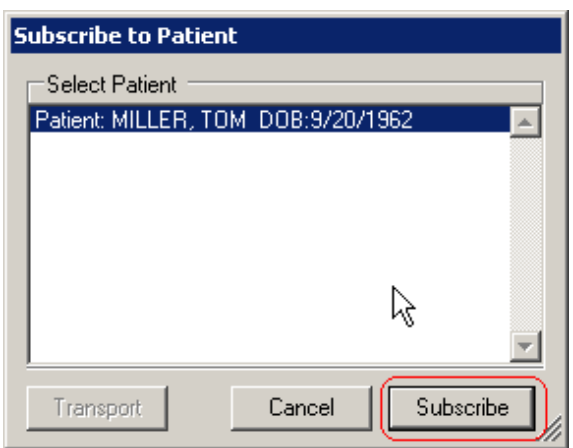

Figure 3. Subscribing to case Tom Miller

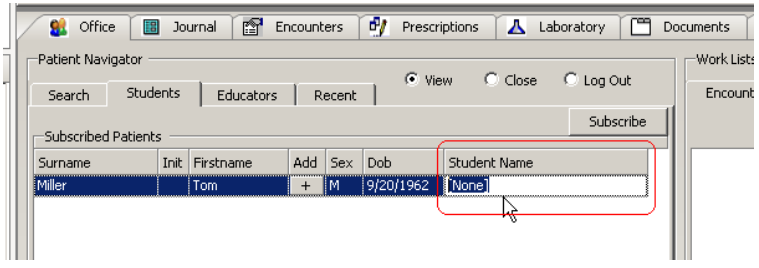

Figure 4. Student subscription screen 
Each user in the EMR system (i.e. each health professional student and each educator) would need to be assigned one of these modes upon creation of a new user account. A user logging into the EMR system will always possess the mode predetermined at the time of account creation. It is intended that each educator and each student would appear as unique users in the system.

The operation of the EMR would then be determined by the mode of the user logged into the system. A small change made to the application software was necessary to filter database queries of the storage system for each user mode. This small change was sufficient to orchestrate and differentiate between these new roles or modes. Resulting in students having access their own patients and educators access their own workspace to create patient cases. Normal or physician users were still able to use their system in their practices all through one application.

Educators would typically create patients or cases and then publish them. Students of newly created accounts would start with an empty workspace and would subscribe to one or more of these publications. When subscribed to a particular patient, all the data that comprises the patient is duplicated into the students account. In effect, the students account holds a private copy of the patient case, separate to that of the educator. This enables the software to maintain a store for each student's actions. Within this framework, the software, therefore, has the capacity to evaluate students either at an individual level or to analyze aggregate responses and possible variations between students.

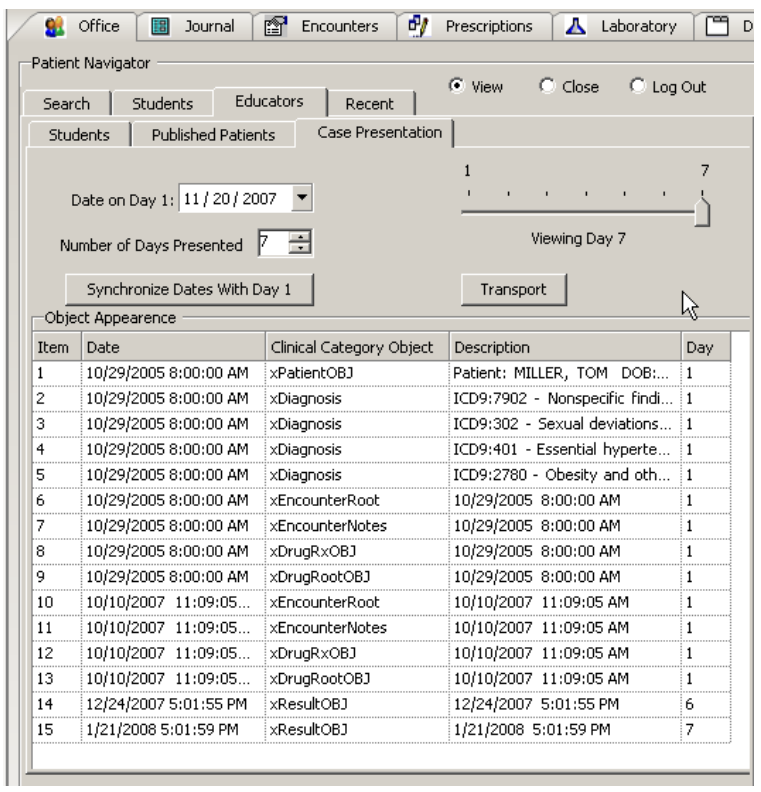

Figure 5. Educator view of Tom Miller. Items 14 and 15, "the results" appear on day 6 and 7 respectively

The concepts of publishing and subscribing is borrowed from database management programs. The EMR, for a typical student login would list the patients available for subscription. When one is chosen from the list (Fig. 3, Fig 4), the entire patients data is copied into the student's private storage space as described above. The ability to subscribe to a patient in this manner allows for an unlimited number of students. For educators, constructing a patient is more involved. In addition to publishing the patient, each object which forms the entire patient record is tagged with an "appearance 
day" attribute. This attribute allows the software to control the day in which each object appears as the case progresses. It may be necessary for certain objects to be hidden from view until their day of appearance (Fig. 5).

For example, tests results and consultation reports based on preceding test orders or referrals respectively would need to be simulated. Therefore, these objects must be hidden from view until at their appropriate time. Constructing a case, therefore, requires a significant amount of planning. An educator's control panel in the software allows the educator to create these objects and set up the "appearance day" attribute for each object. A playback slider conveniently animates the appearance of each object in time sequence by way of a graphical presentation. This same slider also controls the actual staging or appearance of the case as it is being taught in class. Each subscribing student is linked back to appearance slider in this manner, so that every student views the patient case at the appropriate point in time. This feature does not affect the ability for each student to modify their own copy of the subscribed patient case.

In the actual case used for the students, the patient "Tom" was referred for a specialty opinion and the consultation report of the specialist is revealed in the EMR at the appropriate time. Using the above described mechanism, the consultation report therefore, is not accessible to viewing until after the referral is made.

The above method can lead itself to more complex case scenarios by controlling the appearance of certain objects based on certain preceding actions by the student. For example, in a hypothetical respiratory case, a student may order a sputum culture; the sputum result may appear on day four. Alternatively a student ordering a chest x-ray would see the appearance of a typed report on day two. Although different scenarios were not employed in this iteration, the fundamental extensible nature of the EMR allows for more complex scenarios to be built.

There is also a simple mechanism to randomize students to receive the same case but with some variance. For example, using the respiratory case, one set of students may receive a patient complaining of a wet cough and the other set, a dry cough. Objects may also appear by means other than that described above. They can appear based on an interview situation, for example, by asking certain questions, an object representing a response may appear. It is conceived that the flexibility of an object oriented EMR may fulfill the technical requirements of a full medical simulator in the future.

\section{Discussion}

The purpose of the initial implementation was to achieve the delivery of an electronic medical record (EMR) system that could be used for teaching health professional students. The software was unique in that it is not only realistic (being modified from a system currently being used in medical practice) but at the same time it is modifiable from an educational perspective, constituting a unique real-world educational simulation environment. Furthermore, integration of this form of technology into the teaching program of a health professional had not been done in the past at the UBC site or other locations in British Columbia. The risk of failure was significant due to many of the technical glitches which could have occurred with implementing software of this complexity. The initial tested implementation was of a client - server model, however due to the difficulties in obtaining connectivity at all the sites to a central database store, free standing local installations were necessary. There were also difficulties in obtaining 
the hardware for a sophisticated client - server set up including the bandwidth for 200 users potentially accessing the database simultaneously.

To this end, the implementation was successful. The software was stable and did not cause install and execution problems. Preliminary and informal feedback from the students and educators was good. The interface was considered by both groups to be intuitive enough to sit down, to browse and to enter data after the introductory session. Fortunately, technical issues never appeared as a foreground issue. Rather, the intent of the education experience was achieved with the majority of the time spent on discussing the issues of the case and how an information system can document and facilitate care. Thus the project has had two important goals that have been met, namely (1) providing an electronic format for presenting medical cases to be used in education, and (2) at the same time providing health professional students with their first exposure to this important technology which they will be expected to use and master once they graduate. Regarding the second point, it is expected that exposing students to this technology will lead to increased understanding of what it is and how it can be used, and as a consequence will help in increasing the adoption of EMR and electronic health records in general.

The next steps in our work to develop an evaluation framework for student performance as well as a framework to obtain formal feedback in order to improve the software and implementation for future courses.

\section{Conclusion}

It is gratifying to be involved in enriching health professional education and to be able to introduce this new technology into teaching programs as part of overall eHealth initiatives. In this implementation, we satisfactorily introduced an EMR customized for this purpose.

\section{References}

1. Bates, D.W., Teich, J.M., Lee, J., Seger, D., Kuperman, G.J., Ma'Luf, N., et al. (1999). The impact of computerized physician order entry on medication error prevention. Journal of the American Medical Informatics Association, 6(4), 313321.

2. Borycki, E.M., Kushniruk, A.W., Joe, R., Armstrong, B., Otto, T., Ho, K., Silverman, H., Moreau, J. \& Frisch, N. (2009). The University of Victoria Interdisciplinary Electronic Health Record Educational Portal. Studies in Health Technology and Informatics, 143, 49-54.

3. British Columbia eHealth Steering Committee. (2005). eHealth Strategic Framework. (Report November 2005). Retrieved from http://www.health.gov.bc.ca/cpa/publications/ehealth_framework.pdf

4. Canada Health Infoway. (2006). EHRS Blueprint Version 2: Canada Health Infoway, www.infoway.ca.

5. Keenan, C.R., Nguyen, H.H., \& Srinivasan, M. (2006). Electronic medical records and their impact on resident and medical student education. Academic Psychiatry, 30(6), 522-527. 
6. Kohn, L.T., Corrigan, J.M., \& Donaldson, M.S. (2000). To Err is Human: Building a Safer Health System.: Institution of Medicine. National Academy Press, Washington, D.C.

7. Ministry of Health of British Columbia. (2006). eHealth. Retrieved June 6, 2006 from http://www.health.gov.bc.ca/ehealth/index.html.

8. Physician Information Technology Office (PITO). (2007). Frequently asked questions. $\quad$ Retrieved August 20, 2007 from http://www.health.gov.bc.ca/msp/legislation/bcmaagree faqs_pito.html.

9. Romanow, R.G. (2002). Building on values: The future of health care in Canadafinal report. Commission on the future of health care in Canada. (November 2002). Retrieved August 20, 2007 from http://www.hcsc.gc.ca/english/care/romanow/hcc0086.html.

10. Speedie, S.M., \& Niewoehner, C. (2003). The Minnesota Virtual Clinic: using a simulated EMR to teach medical students basic science and clinical concepts. AMIA Annual Symposium Proceedings/AMIA Symposium, 1013.

11. Zelnick, C.J., \& Nelson, D.A. (2002). A medical informatics curriculum for $21 \mathrm{st}$ century family practice residencies. Family Medicine, 34(9), 685-691. 